\section{P93 FAECAL MICROBIOTA TRANSPLANT FOR REFRACTORY CHECKPOINT INHIBITOR IMMUNOTHERAPY-RELATED COLITIS}

${ }^{1}$ Vincent Cheung*, ${ }^{1}$ Sarah Sasson, ${ }^{2,3}$ Mariya Misheva, ${ }^{1}$ Stephanie Slevin, ${ }^{1}$ Tarun Gupta, ${ }^{1}$ Gwilym Webb, ${ }^{1}$ Alissa Walsh, ${ }^{1}$ Alison Simmons, ${ }^{4}$ Nabil Quraishi, ${ }^{4}$ Tariq Iqbal, ${ }^{5}$ Mark Middleton, ${ }^{5}$ Benjamin Fairfax, ${ }^{5}$ Miranda Payne, ${ }^{3}$ James McCullagh, ${ }^{1}$ Paul Klenerman, ${ }^{1}$ Oliver Brain. ${ }^{1}$ Translational Gastroenterology Unit, Oxford, UK; ${ }^{2}$ Oxford Centre for Microbiome Studies, Oxford, UK; ${ }^{3}$ Chemistry Research Laboratory, Oxford, UK; ${ }^{4}$ Microbiome Treatment Centre, Birmingham, UK; ${ }^{5}$ Oxford Cancer Centre, Oxford, UK

\subsection{6/gutjnl-2020-bsgcampus. 168}

Introduction Immune checkpoint inhibitors (ICIs) have revolutionised the treatment of various cancers. They improve survival but this comes at the cost of immune-related adverse events like ICI-colitis. First line treatment is steroids and refractory cases can be treated with infliximab, vedolizumab or faecal microbiota transplant (FMT). We describe our experience of FMT, alongside cytometric/transcriptomic analysis of the GI immune response, and whole stool metabolomic analysis pre- and post-FMT.

Methods $150 \mathrm{ml}$ FMT was delivered to the caecum by colonoscopy. 5 pairs of colon pinch biopsies were collected pre- and week 6 post-FMT. Gut mononuclear cells (GMNC) were isolated from the biopsies by enzymatic/mechanical digestion. GMNC were analysed using a 21-parameter flow cytometry panel on the Cytek Aurora Spectral Analyser and a 780-plex Nanostring panel. Stool pre- and post-FMT, and that of the FMT donor, was subjected to whole metabolome analysis.

Results Two patients with refractory ICI-colitis were treated with FMT (same donor). Characteristics are shown in Table 1:

Patient 1's symptoms of diarrhoea and abdominal pain resolved fully. Week 6 sigmoidoscopy and biopsies were normal. Pre-FMT, there were high levels of activation (as measured by co-expression of HLA-DR and CD38) on CD4, CD8 and MAIT cell subsets as well as high $\mathrm{Ki}-67$ and low expression of Bcl-2. Post FMT, there was reduced expression of HLA-DR and CD38 and lower expression of Ki-67 with high/ homeostatic levels of $\mathrm{Bcl}-2$ and an increased proportion of Tregs.

The bowel frequency of patient 2 demonstrated some clinical improvement, but week 6 sigmoidoscopy showed an unchanged UCEIS score and Nancy histological index. Pre FMT, there were similar levels of activation as seen in patient 1 , but post-FMT responses were muted.

We will present an analysis of the GI immune response and of the stool whole metabolome, in these two patients with dichotomous clinical outcomes.

Conclusions We report the first use of FMT to treat ICI-colitis in the UK, demonstrating that it is effective in a subset of patients. Although both patients received stool from the same

\begin{tabular}{|c|c|c|c|c|c|c|c|}
\hline Patient & Age & Sex & Cancer & ICI & $\begin{array}{l}\text { ICI-colitis } \\
\text { treatment } \\
\text { before FMT }\end{array}$ & $\begin{array}{l}\text { UCEIS } \\
\text { pre } \\
\text { FMT }\end{array}$ & $\begin{array}{l}\text { Nancy } \\
\text { index } \\
\text { pre FMT }\end{array}$ \\
\hline 1 & 63 & $\mathrm{~F}$ & Melanoma & $\begin{array}{l}\text { Ipilimumab/ } \\
\text { nivolumab }\end{array}$ & $\begin{array}{l}\text { Steroids, } \\
\text { infliximab, } \\
\text { vedolizumab }\end{array}$ & 4 & 3 \\
\hline 2 & 61 & M & Lung & Pembrolizumab & $\begin{array}{l}\text { Steroids, } \\
\text { infliximab }\end{array}$ & 5 & 3 \\
\hline
\end{tabular}

donor, they experienced contrasting treatment responses. We will present the salient GI immune and stool metabolomic differences to reveal mechanistic insights.

\section{P94 VEDOLIZUMAB FOR INFLAMMATORY BOWEL DISEASE IN PRE AND POST LIVER TRANSPLANT}

Yooyun Chung ${ }^{*}$, Shanika Nayagam, Guy Chung-Faye, Patrick Dubois, Bu'Hussain Hayee, Lucy Medcalf, Michael Heneghan, Deepak Joshi, Alexandra Kent. King's College Hospital NHS Trust, London, UK

\subsection{6/gutjnl-2020-bsgcampus. 169}

Introduction The association between inflammatory bowel disease (IBD) and autoimmune liver disease (AILD) is well established. Vedolizumab (VDZ) is a gut-specific anti- $\alpha 4 \beta 7$ integrin antibody used to treat IBD. VDZ is favoured for AILD patients on additional immunosuppression. This retrospective observational study at a tertiary liver transplant centre aims to review VDZ use in IBD-AILD pre and post liver transplant (LT).

Method An electronic database of IBD patients treated with VDZ from November 2014 to February 2020 was used to identify those with AILD. Data on the nature of AILD, response to VDZ, complications or reason for cessation of VDZ was recorded.

Results 36 patients with IBD-AILD have been treated with VDZ, with 18 patients receiving ongoing therapy. Demographics are shown in table 1.

Of the 18 pre-LT patients, 1 had septic shock from cholangitis at the onset of VDZ therapy, 1 was diagnosed with breast cancer and cholangiocarcinoma a month after VDZ cessation for primary non response. 2 patients underwent LT whilst on VDZ with no post-operative complications.

Of the 18 post-LT patients, several complications were seen. There was a single case of: recurrent pharyngitis,

Abstract P94 Table 1 Vedolizumab (VDZ), Ulcerative colitis (UC), Crohn's disease (CD), IBD-unspecified (IBDU), autoimmune sclerosing cholangitis (AISC), primary sclerosing cholangitis (PSC), autoimmune hepatitis (AlH)

\begin{tabular}{|c|c|c|}
\hline & Current VDZ & Discontinued VDZ \\
\hline Number & 18 & 18 \\
\hline \multicolumn{3}{|l|}{ Gender } \\
\hline Female & 4 & 5 \\
\hline Male & 14 & 13 \\
\hline \multicolumn{3}{|c|}{ Age: years } \\
\hline Mean & 31.6 & 37.2 \\
\hline Range & $19-62$ & $18-71$ \\
\hline \multicolumn{3}{|c|}{ Diagnosis } \\
\hline UC & 13 & 14 \\
\hline$C D$ & 3 & 2 \\
\hline IBDU & 2 & 2 \\
\hline \multicolumn{3}{|c|}{ Duration of treatment: days } \\
\hline Mean & 540 & 345 \\
\hline Range & 8-1241 & $41-1024$ \\
\hline \multicolumn{3}{|c|}{ Liver disease } \\
\hline AISC & 3 & 3 \\
\hline PSC & 5 & 3 \\
\hline AlH & 1 & 3 \\
\hline OLT & 9 & 9 \\
\hline
\end{tabular}

